\title{
Dynamics of almost periodic solutions for a discrete Fox harvesting model with feedback control
}

\section{Jehad Alzabut ${ }^{*}$}

\section{"Correspondence:} jalzabut@psu.edu.sa Department of Mathematics and Physical Sciences, Prince Sultan University, P.O. Box 66833, Riyadh, 11586, Saudi Arabia

\begin{abstract}
We consider the following discrete Fox harvesting model with feedback control of the form

$$
\left\{\begin{array}{l}
x(n+1)=x(n) \exp \left\{\beta(n) \operatorname{In}^{r}\left(\frac{k(n)}{x(n)}\right)-\alpha(n)-\gamma(n) u(n)\right\}, \\
\Delta u(n)=-\mu(n) u(n)+v(n) x(n) .
\end{array}\right.
$$

Under the assumptions of almost periodicity of the coefficients, sufficient conditions are established for the existence and uniformly asymptotical stability of almost periodic solutions of this model. The persistence as well as the boundedness of solutions of the above system are discussed prior to presenting the main result. Examples are provided to illustrate the effectiveness of the proposed results.
\end{abstract}

MSC: 39A11;34K14

Keywords: discrete Fox harvesting model; almost periodic; persistence and boundedness; feedback control; uniformly asymptotical stability

\section{Introduction}

Consider the following equation of population dynamics [1, 2]:

$$
x^{\prime}(t)=-x F(t, x)+x G(t, x), \quad x^{\prime}(t)=\frac{\mathrm{d} x}{\mathrm{~d} t},
$$

where $x=x(t)$ is the size of the population, $F(t, x)$ is the per-capita harvesting rate and $G(t, x)$ is the per-capita fecundity rate. Let $G(t, x)$ and $F(t, x)$ be defined in the form

$$
F(t, x)=\alpha(t) \quad \text { and } \quad G(t, x)=\beta(t) \ln ^{r}\left(\frac{K(t)}{x(t)}\right), \quad r>0,
$$

then equation (1) becomes

$$
x^{\prime}(t)=x(t)\left[\beta(t) \ln ^{r}\left(\frac{K(t)}{x(t)}\right)-\alpha(t)\right]
$$

where $\alpha(t)$ is a variable harvesting rate, $\beta(t)$ is an intrinsic factor and $K(t)$ is a varying environmental carrying capacity. The positive parameter $r$ is referred to as an interaction parameter $[1,3,4]$. Indeed, if $r>1$ then intra-specific competition is high, whereas

\section{然 Springer}

(c) 2012 Alzabut; licensee Springer. This is an Open Access article distributed under the terms of the Creative Commons Attribution License (http://creativecommons.org/licenses/by/2.0), which permits unrestricted use, distribution, and reproduction in any medium, provided the original work is properly cited. 
if $0<r<1$, then the competition is low. For $r=1$, equation (2) reduces to the classical Gompertzian model with harvesting [2, 5]. Equation (2) is called a Fox surplus production model that has been used to build up certain prediction models such as microbial growth model, demographic model and fisheries model. This equation is considered to be an efficient alternative to the well known $r$-logistic model. Specifically, the Fox model is more appropriate upon describing lower population density; we refer the reader to $[1,3$, $4,6-10]$ and, in particular, to the recent paper [11] for more information.

Ecosystems in the real world are continuously disturbed by unpredictable forces which can result in changing some biological parameters such as survival rates. In ecology, a question of practical interest is whether or not an ecosystem can withstand these unpredictable disturbances which persist for a finite period of time. In the language of control theory, we call these disturbance functions a control variable. In [12], Gopalsamy and Weng introduced a model with feedback controls in which the control variables satisfy a certain differential equation. The next years have witnessed the appearance of many papers regarding the study of ecosystems with feedback control; see for instance [13-17].

In the last years, many authors have argued that the discrete time models governed by difference equations are more appropriate than the continuous counterparts, especially when the populations have no overlapping generations. It is also known that the discrete models can provide more efficient computational methods for numerical simulations [1820]. By applying the same method used in [21], one can derive the discrete analogue of (2) as follows:

$$
x(n+1)=x(n) \exp \left\{\beta(n) \ln ^{r}\left(\frac{K(n)}{x(n)}\right)-\alpha(n)\right\} .
$$

One of the most important behaviors of solutions which has been the main object of investigations among authors is the periodic behavior of solutions [22-29]. To consider periodic environmental factors acting on a population model, it is natural to study the model subject to periodic coefficients. Indeed, the assumption of periodicity of the parameters in the model is a way of incorporating the time-dependent variability of the environment (e.g., seasonal effects of weather, food supplies, mating habits and harvesting). On the other hand, upon considering long-term dynamical behavior, it has been found that the periodic parameters often turn out to experience some perturbations that may lead to a change in character. Thus, the investigation of almost periodic behavior is considered to be in more accordance with reality; see the remarkable monographs [30-32] and the recent contributions [33-48].

Motivated by the above justifications, we consider the following discrete Fox harvesting model with feedback control in the form

$$
\left\{\begin{array}{l}
x(n+1)=x(n) \exp \left\{\beta(n) \ln ^{r}\left(\frac{K(n)}{x(n)}\right)-\alpha(n)-\gamma(n) u(n)\right\}, \\
\Delta u(n)=-\mu(n) u(n)+v(n) x(n)
\end{array}\right.
$$

where $u(n)$ is the control variable and $\Delta u(n)$ is the forward difference $u(n+1)-u(n)$. Under the assumptions of almost periodicity of coefficients of system (4), we shall study the existence and uniformly asymptotical stability of almost periodic solutions for system (4). The persistence as well as the boundedness of solutions of system (4) are discussed prior to presenting the main result. To the best of author's observation, no paper has been 
published in the literature regarding the dynamics of almost periodic solutions of system (4). Thus, the result of this paper is essentially different and presents a new approach.

The remaining part of this paper is organized as follows. In Section 2, some preliminary definitions along with essential lemmas are given. Section 3 discusses the persistence and boundedness of solutions of system (4). In Section 4, sufficient conditions are established to investigate the existence and uniformly asymptotical stability of almost periodic solutions of the said system. Section 5 provides some numerical examples to illustrate the feasibility of our theoretical results.

\section{Preliminaries}

Let $\mathbb{R}, \mathbb{R}^{+}, \mathbb{Z}$ and $\mathbb{Z}^{+}$be the sets of real, nonnegative real, integer and nonnegative integer numbers respectively. For any bounded sequence $\{f(n)\}$ on $\mathbb{Z}$, we define

$$
f^{M}=\sup _{n \in \mathbb{Z}} f(n) \quad \text { and } \quad f^{m}=\inf _{n \in \mathbb{Z}} f(n)
$$

Throughout the remainder of this paper, we assume the following condition:

(H.1) $\{\beta(n)\},\{K(n)\},\{\alpha(n)\},\{\gamma(n)\},\{\mu(n)\}$ and $\{v(n)\}$ are bounded nonnegative almost periodic sequences such that

$$
\begin{array}{llc}
0<\beta^{m} \leq \beta(n) \leq \beta^{M}, & 0<K^{m} \leq K(n) \leq K^{M}, & 0<\alpha^{m} \leq \alpha(n) \leq \alpha^{M}, \\
0<\gamma^{m} \leq \gamma(n) \leq \gamma^{M}, & 0<v^{m} \leq v(n) \leq v^{M}, & 0<\mu^{m} \leq \mu(n) \leq \mu^{M}<1 .
\end{array}
$$

Due to certain biological reasons, we restrict our attention to positive solutions of system (4). Thus, we consider system (4) together with the following initial conditions:

$$
x(0)>0 \text { and } u(0)>0 .
$$

One can easily figure out that the solutions of system (4) with the initial conditions (6) are defined and remain positive for all $n \in \mathbb{Z}^{+}$.

Definition 1 [49] A sequence $x: \mathbb{Z} \rightarrow \mathbb{R}$ is called an almost periodic sequence if the $\epsilon$ translation set $E\{\epsilon, x\}=\{\tau \in \mathbb{Z}: \mid x(n+\tau)-x(n)<\epsilon, \forall n \in \mathbb{Z}\}$ is a relatively dense set in $\mathbb{Z}$ for all $\epsilon>0$, that is, for any $\epsilon>0$ there is a constant $l(\epsilon)>0$ such that in any interval of length $l(\epsilon)$ there exists a number $\tau \in E\{\epsilon, x\}$ such that the inequality

$$
|x(n+\tau)-x(n)|<\epsilon
$$

is satisfied for all $n \in \mathbb{Z}$.

Definition 2 [49] Let $f: \mathbb{Z} \times \mathbb{D} \rightarrow \mathbb{R}$ where $D$ is an open set in $\mathbb{R}$. Then $f(n, x)$ is said to be almost periodic in $n$ uniformly for $x \in \mathbb{D}$, or uniformly almost periodic for short, if for any $\epsilon>0$ and any compact set $\mathbb{S} \in \mathbb{D}$, there exists a positive integer $l(\epsilon, \mathbb{S})$ such that any interval of length $l(\epsilon, \mathbb{S})$ contains an integer $\tau$ for which

$$
|f(n+\tau, x)-f(n, x)|<\epsilon
$$

for all $n \in \mathbb{Z}$ and $x \in \mathbb{S}$. The number $\tau$ is called the $\epsilon$-translation for $f(n, x)$. 
Lemma 1 [49] $\{x(n)\}$ is an almost periodic sequence if and only iffor any sequence $\left\{h_{k}^{\prime}\right\} \subset$ $\mathbb{Z}$, there exists a subsequence $\left\{h_{k}\right\} \subset\left\{h_{k}^{\prime}\right\}$ such that $x\left(n+h_{k}\right)$ converges uniformly on $n \in \mathbb{Z}$ as $k \rightarrow \infty$. Furthermore, the limit sequence is also an almost periodic sequence.

Consider the following almost periodic difference system:

$$
x(n+1)=f(n, x(n)), \quad n \in \mathbb{Z}^{+},
$$

where $f: \mathbb{Z} \times \mathbb{S}_{\delta} \rightarrow \mathbb{R}, \mathbb{S}_{\delta}=\{x \in \mathbb{R}:\|x\|<\delta\}$ and $f(n, x)$ is almost periodic in $n$ uniformly for $x \in \mathbb{S}_{\delta}$ and is continuous in $x$. The product system of (7) is in the form

$$
x(n+1)=f(n, x(n)), \quad y(n+1)=f(n, y(n)) .
$$

Our approach is based on the following lemma.

Lemma 2 [50] Suppose that there exists a Lyapunov functional $V(n, x, y)$ defined for $n \in$ $\mathbb{Z}^{+},\|x\|<\delta,\|y\|<\delta$ and satisfying the following conditions:

(i) $a(\|x-y\|) \leq V(n, x, y) \leq b(\|x-y\|)$, where $a, b \in T$ with $T=\left\{a \in C\left(\mathbb{R}^{+}, \mathbb{R}^{+}\right): a(0)=0\right.$ and $a$ is increasing $\}$

(ii) $\left|V\left(n, x_{1}, y_{1}\right)-V\left(n, x_{2}, y_{2}\right)\right| \leq L\left(\left\|x_{1}-x_{2}\right\|+\left\|y_{1}-y_{2}\right\|\right)$ where $L>0$ is a constant;

(iii) $\Delta_{(8)} V(n, x, y) \leq-\lambda V(n, x, y)$ where $0<\lambda<1$ is a constant and $\Delta_{(8)} V(n, x, y)=V(n+1, f(n, x), f(n, y))-V(n, x, y)$.

Moreover, if there exists a solution $\varphi(n)$ of system (8) such that $\|\varphi(n)\| \leq \delta^{*}<\delta$ for $n \in \mathbb{Z}^{+}$, then there exists a unique uniformly asymptotically stable almost periodic solution $p(n)$ of system (8) which satisfies $\|p(n)\| \leq \delta^{*}$. In particular, if $f(n, x)$ is periodic of period $\omega$, then there exists a unique uniformly asymptotically stable periodic solution of system (8) of period $\omega$.

\section{Persistence and boundedness}

In this section, we prove every solution of system (4) is persistent. In addition to this, we prove that there exists a bounded solution for (4).

Definition 3 System (4) is said to be persistent if there are positive constants $x^{*}, u^{*}, x_{*}$ and $\mathcal{u}$ * such that

$$
\limsup _{n \rightarrow \infty} x(n) \leq x^{*}, \quad \limsup _{n \rightarrow \infty} u(n) \leq u^{*}
$$

and

$$
\liminf _{n \rightarrow \infty} x(n) \geq x_{*}, \quad \limsup _{n \rightarrow \infty} u(n) \geq u_{*}
$$

for each positive solution $(x(n), u(n))$ of $(4)$.

We assume the following condition:

(H.2) $\beta^{m} \ln ^{r} K^{m}>\alpha^{M}+\gamma^{M} u^{*}$. 
Set

$$
x^{*}=K^{M} e^{\left[\left(\frac{\alpha}{\beta}\right)^{m}\right]^{-\frac{1}{r}}} \exp \left\{\left(\beta \ln ^{r} K\right)^{M}\right\} \quad \text { and } \quad u^{*}=\frac{v^{M} x^{*}}{\mu^{m}} .
$$

Lemma 3 Let (H.1), (H.2) hold. Then, every solution of system (4) satisfies

$$
\limsup _{n \rightarrow \infty} x(n) \leq x^{*} \quad \text { and } \quad \limsup _{n \rightarrow \infty} u(n) \leq u^{*}
$$

Proof Let $(x(n), u(n))$ be a solution of (4). To prove that $\lim _{\sup _{n \rightarrow \infty}} x(n) \leq x^{*}$, we consider two cases:

Case I. There exists $l_{0} \in \mathbb{Z}^{+}$such that $x\left(l_{0}+1\right) \geq x\left(l_{0}\right)$. By the first equation of (4), we have

$$
\beta\left(l_{0}\right) \ln ^{r}\left(\frac{K\left(l_{0}\right)}{x\left(l_{0}\right)}\right)-\alpha\left(l_{0}\right)-\gamma\left(l_{0}\right) u\left(l_{0}\right) \geq 0,
$$

which implies that

$$
x\left(l_{0}\right) \leq K^{M} e^{\left[\left(\frac{\alpha}{\beta}\right)^{m}\right]^{-\frac{1}{r}}} \leq x^{*} .
$$

Thus,

$$
\begin{aligned}
x\left(l_{0}+1\right) & =x\left(l_{0}\right) \exp \left\{\beta\left(l_{0}\right) \ln ^{r}\left(\frac{K\left(l_{0}\right)}{x\left(l_{0}\right)}\right)-\alpha\left(l_{0}\right)-\gamma\left(l_{0}\right) u\left(l_{0}\right)\right\} \\
& \leq K^{M} e^{\left[\left(\frac{\alpha}{\beta}\right)^{m}\right]^{-\frac{1}{r}}} \exp \left\{\left(\beta \ln ^{r} K\right)^{M}\right\}=x^{*} .
\end{aligned}
$$

We claim that $x(n) \leq x^{*}$ for $n \geq l_{0}$. Indeed, if there is an integer $n_{0} \geq l_{0}+2$ such that $x\left(n_{0}\right)>$ $x^{*}$ and $n_{1}$ is the least integer between $l_{0}$ and $n_{0}$ such that $x\left(n_{1}\right)=\max _{l_{0} \leq n \leq n_{0}}\{x(n)\}$, then $n_{1} \geq l_{0}+2$ and $x\left(n_{1}\right)>x\left(n_{1}-1\right)$ which implies that $x\left(n_{1}\right) \leq x^{*}<x\left(n_{0}\right)$. This is a contradiction. This proves the claim.

Case II. Let $x(n) \geq x(n+1)$ for $n \in \mathbb{Z}^{+}$. Then, $\lim _{n \rightarrow \infty} x(n)$ exists and equals $\bar{x}$. Taking the limit of the first equation in (4), we have

$$
\lim _{n \rightarrow \infty}\left(\beta(n) \ln ^{r}\left(\frac{K(n)}{x(n)}\right)-\alpha(n)-\gamma(n) u(n)\right)=0 .
$$

Hence $\bar{x} \leq K^{M} e^{\left[\left(\frac{\alpha}{\beta}\right)^{m}\right]^{-\frac{1}{r}}} \leq x^{*}$. This proves the claim.

Now, we prove that $\limsup _{n \rightarrow \infty} u(n) \leq u^{*}$. For any $\varepsilon>0$, there exists a large enough integer $n_{0} \in \mathbb{Z}^{+}$such that $x(n) \leq x^{*}+\varepsilon$ for $n \geq n_{0}$. By the second equation of (4), we get

$$
\begin{aligned}
u(n) & =\prod_{i=0}^{n-1}(1-\mu(i))\left[u(0)+\sum_{i=0}^{n-1} \frac{v(i) x(i)}{\prod_{j=0}^{i}(1-\mu(j))}\right] \\
& \leq\left(1-\mu^{m}\right)^{n}\left[u(0)+\sum_{i=0}^{n_{0}-1} \frac{v(i) x(i)}{\prod_{j=0}^{i}(1-\mu(j))}\right]+v^{M}\left(x^{*}+\varepsilon\right) \sum_{i=n_{0}}^{n-1} \prod_{j=i-1}^{n-1}(1-\mu(j))
\end{aligned}
$$




$$
\begin{aligned}
& \leq\left(1-\mu^{m}\right)^{n}\left[u(0)+\sum_{i=0}^{n_{0}-1} \frac{v(i) x(i)}{\prod_{j=0}^{i}(1-\mu(j))}\right] \\
& +v^{M}\left(x^{*}+\varepsilon\right) \sum_{i=n_{0}}^{n-1}\left(1-\mu^{m}\right)^{n-i-1} .
\end{aligned}
$$

Since $0<\mu^{m}<1$, we can find a positive number $d$ such that $1-\mu^{m}=e^{-d}$. Thus, by using Stolz's theorem, we obtain

$$
\lim _{n \rightarrow \infty} \sum_{i=n_{0}}^{n-1}\left(1-\mu^{m}\right)^{n-i-1}=\lim _{n \rightarrow \infty} \frac{\sum_{i-n_{0}}^{n-1} e^{d(i+1)}}{e^{d n}}=\frac{1}{1-e^{-d}}=\frac{1}{\mu^{m}} .
$$

Hence

$$
\limsup _{n \rightarrow \infty} u(n) \leq \frac{v^{M}\left(x^{*}+\varepsilon\right)}{\mu^{m}}
$$

By the arbitrariness of $\varepsilon$, we obtain $\lim \sup _{n \rightarrow \infty} u(n) \leq \frac{v^{M} x^{*}}{\mu^{m}}:=u^{\prime \prime}$. The proof of Lemma 3 is complete.

Set

$$
x_{*}=K^{m} e^{\left(\frac{\alpha^{M}+\gamma^{M}\left(u^{*}\right)}{\beta^{m}}\right)^{-\frac{1}{r}}} \exp \left\{\beta^{m} \ln ^{r}\left(\frac{K^{m}}{x^{*}}\right)-\alpha^{M}-\gamma^{M} u^{*}\right\} \quad \text { and } \quad u_{*}=\frac{v^{m} x^{*}}{\mu^{M}} .
$$

Lemma 4 Let (H.1), (H.2) hold. Then every solution of system (4) satisfies

$$
\liminf _{n \rightarrow \infty} x(n) \geq x_{*} \text { and } \quad \liminf _{n \rightarrow \infty} u(n) \geq u_{*}
$$

Proof Let $(x(n), u(n))$ be a solution of (4). By virtue of Lemma 3, one can figure out that for any $\varepsilon>0$ which satisfies $\beta^{m} \ln ^{r} K^{m}>\alpha^{M}+\gamma^{M}\left(u^{*}+\varepsilon\right)$, there exists $n_{0} \in \mathbb{Z}^{+}$such that

$$
x(n) \leq x^{*}+\varepsilon \quad \text { and } \quad u(n) \leq u^{*}+\varepsilon \quad \text { for } n \geq n_{0} .
$$

To prove that $\liminf _{n \rightarrow \infty} x(n) \geq x^{*}$, we consider two cases:

Case I. There exists $l_{0} \geq n_{0}$ such that $x\left(l_{0}+1\right) \leq x\left(l_{0}\right)$. We observe that for $n \geq l_{0}$, we have

$$
\begin{aligned}
x(n+1) & =x(n) \exp \left\{\beta(n) \ln ^{r}\left(\frac{K(n)}{x(n)}\right)-\alpha(n)-\gamma(n) u(n)\right\} \\
& \geq x(n) \exp \left\{\beta^{m} \ln ^{r}\left(\frac{K(n)}{x(n)}\right)-\alpha^{M}-\gamma^{M}\left(u^{*}+\varepsilon\right)\right\} .
\end{aligned}
$$

For $n=l_{0}$, we get

$$
\beta^{m} \ln ^{r}\left(\frac{K\left(l_{0}\right)}{x\left(l_{0}\right)}\right)-\alpha^{M}-\gamma^{M}\left(u^{*}+\varepsilon\right) \leq 0
$$


which implies that

$$
x\left(l_{0}\right) \geq K^{m} e^{\left(\frac{\alpha^{M}+\gamma^{M}\left(u^{*}+\varepsilon\right)}{\beta^{m}}\right)^{-\frac{1}{r}}} .
$$

It follows that

$$
\begin{aligned}
x\left(l_{0}+1\right) & =x\left(l_{0}\right) \exp \left\{\beta\left(l_{0}\right) \ln ^{r}\left(\frac{K\left(l_{0}\right)}{x\left(l_{0}\right)}\right)-\alpha\left(l_{0}\right)-\gamma\left(l_{0}\right) u\left(l_{0}\right)\right\} \\
& \geq K^{m} e^{\left(\frac{\alpha^{M}+\gamma^{M}\left(u^{*}+\varepsilon\right)}{\beta^{m}}\right)^{-\frac{1}{r}}} \exp \left\{\beta^{m} \ln ^{r}\left(\frac{K^{m}}{x^{\prime \prime}+\varepsilon}\right)-\alpha^{M}-\gamma^{M}\left(u^{*}+\varepsilon\right)\right\} .
\end{aligned}
$$

Let

$$
x_{\varepsilon}=K^{m} e^{\left(\frac{\alpha^{M}+\gamma^{M}\left(u^{*}+\varepsilon\right)}{\beta^{m}}\right)^{-\frac{1}{r}}} \exp \left\{\beta^{m} \ln ^{r}\left(\frac{K^{m}}{x^{*}+\varepsilon}\right)-\alpha^{M}-\gamma^{M}\left(u^{*}+\varepsilon\right)\right\} .
$$

We claim that

$$
x(n) \geq x_{\varepsilon} \quad \text { for } n \geq l_{0} .
$$

For the sake of contradiction, assume that there exists $p_{0} \geq l_{0}$ such that $x\left(p_{0}\right)<x_{\varepsilon}$. Then $p_{0} \geq l_{0}+2$. Let $p_{1} \geq l_{0}+2$ be the smallest integer such that $x\left(p_{1}\right)<x_{\varepsilon}$. Then $x\left(p_{1}-1\right)>x\left(p_{1}\right)$. The above arguments imply that $x\left(p_{1}\right) \geq x_{\varepsilon}$ which is a contradiction. This proves the claim.

Case II. Let $x(n)>x(n+1)$ for all $n \in \mathbb{Z}^{+}$. Then, $\lim _{n \rightarrow \infty} x(n)$ exists and it is equal to $\underline{x}$. Taking the limit of the first equation of (4), we have

$$
\lim _{n \rightarrow \infty}\left(\beta(n) \ln ^{r}\left(\frac{K(n)}{x(n)}\right)-\alpha(n)-\gamma(n) u(n)\right)=0 .
$$

Hence $\underline{x} \geq K^{m} e^{\left(\frac{\alpha^{M}+\gamma^{M}\left(u^{*}+\varepsilon\right)}{\beta^{m}}\right)^{-\frac{1}{r}}} \geq x_{\varepsilon}$ and $\lim _{\varepsilon \rightarrow 0} x_{\varepsilon}=x_{*}$. This proves the claim.

By applying the same arguments followed in the proof of Lemma 3 , one can easily show that $\lim _{n \rightarrow \infty} u(n) \geq u *$. The proof of Lemma 4 is complete.

The results of Lemma 3 and Lemma 4 can be concluded in the following theorem:

Theorem 1 Let (H.1), (H.2) hold. Then system (4) is persistent.

Let $\Omega$ be the set of all solutions $(x(n), u(n))$ of system (4) satisfying $x=x(n) \leq x^{*}$ and $u^{*} \leq u(n) \leq u^{*}$ for all $n \in \mathbb{Z}^{+}$. By virtue of Theorem 1 , it should be noted that $\Omega$ is an invariant set of system (4).

In view of Lemma 2, we need to show that there exists a bounded solution of system (4). The following result proves the existence of such a solution.

Theorem 2 Let (H.1), (H.2) hold. Then $\Omega \neq \emptyset$.

Proof By the almost periodicity of $\{\beta(n)\},\{K(n)\},\{\alpha(n)\},\{\gamma(n)\},\{\mu(n)\}$ and $\{v(n)\}$, there exists an integer valued sequence $\left\{\tau_{p}\right\}$ with $\left\{\tau_{p}\right\} \rightarrow \infty$ as $p \rightarrow \infty$ such that $\beta\left(n+\tau_{p}\right) \rightarrow$ $\beta(n), K\left(n+\tau_{p}\right) \rightarrow K(n), \alpha\left(n+\tau_{p}\right) \rightarrow \alpha(n), \gamma\left(n+\tau_{p}\right) \rightarrow \gamma(n), \mu\left(n+\tau_{p}\right) \rightarrow \mu(n), v\left(n+\tau_{p}\right) \rightarrow$ 
$v(n)$ as $p \rightarrow \infty$. Let $\varepsilon$ be an arbitrary small positive number. It follows from Lemma 3 and Lemma 4 that there exists a positive integer $N_{0}$ such that

$$
x *-\varepsilon \leq x(n) \leq x^{*}+\varepsilon \quad \text { and } \quad u *-\varepsilon \leq u(n) \leq u^{*}+\varepsilon, \quad \text { for all } n>N_{0} .
$$

Let $x_{p}(n)=x\left(n+\tau_{p}\right)$ and $u_{p}(n)=u\left(n+\tau_{p}\right)$ for $n \geq N_{0}-\tau_{p}, p=1,2, \ldots$. For any positive integer $q$, it is easy to see that there exist sequences $\left\{x_{p}(n): p \geq q\right\}$ and $\left\{u_{p}(n): p \geq q\right\}$ such that the sequences $\left\{x_{p}(n)\right\}$ and $\left\{u_{p}(n)\right\}$ have subsequences, denoted by $\left\{x_{p}(n)\right\}$ and $\left\{u_{p}(n)\right\}$ again, converging on any finite interval of $\mathbb{Z}$ as $p \rightarrow \infty$, respectively. Thus, we have sequences $\{z(n)\}$ and $\{w(n)\}$ such that

$$
x_{p}(n) \rightarrow z(n) \quad \text { and } \quad u_{p}(n) \rightarrow w(n) \quad \text { for } n \in \mathbb{Z} \text { as } p \rightarrow \infty .
$$

Therefore, the system

$$
\left\{\begin{array}{l}
x_{p}(n+1)=x_{p}(n) \exp \left\{\beta\left(n+\tau_{p}\right) \ln ^{r}\left(\frac{K\left(n+\tau_{p}\right)}{x_{p}(n)}\right)-\alpha\left(n+\tau_{p}\right)-\gamma\left(n+\tau_{p}\right) u_{p}(n)\right\}, \\
u_{p}(n+1)=\left(1-\mu\left(n+\tau_{p}\right)\right) u_{p}(n)+v\left(n+\tau_{p}\right) x_{p}(n),
\end{array}\right.
$$

implies

$$
\left\{\begin{array}{l}
z(n+1)=z(n) \exp \left\{\beta(n) \ln ^{r}\left(\frac{K(n)}{z(n)}\right)-\alpha(n)-\gamma(n) w(n)\right\} \\
w(n)=(1-\mu(n)) w(n)+v(n) z(n)
\end{array}\right.
$$

We can easily see that $(z(n), w(n))$ is a solution of system (4) and $x *-\varepsilon \leq z(n) \leq x^{*}+\varepsilon$, $u^{*}-\varepsilon \leq w(n) \leq u^{*}+\varepsilon$ for $n \in \mathbb{Z}$. Since $\varepsilon$ is arbitrary, it follows that $x^{*} \leq z(n) \leq x^{*}, u^{*} \leq$ $w(n) \leq u^{*}$ for $n \in \mathbb{Z}$. This completes the proof.

\section{The main result}

Let $f(x)=\ln ^{r}\left(\frac{K(n)}{e^{x}}\right)$, where $x \in\left[\ln x_{*}, \ln x^{*}\right]$. Then, it is easy to find out that $f^{\prime}(x)=$ $-r\left[\ln \left(\frac{K(n)}{e^{x}}\right)\right]^{r-1}$. The following inequalities hold:

$$
-r\left[\ln \left(\frac{K(n)}{e^{x}}\right)\right]^{r-1} \leq-r\left[\ln \left(\frac{K^{m}}{x^{*}}\right)\right]^{r-1}:=\Phi_{1}
$$

and

$$
r\left[\ln \left(\frac{K(n)}{e^{x}}\right)\right]^{r-1} \leq r \ln ^{r-1} K^{M}:=\Phi_{2}
$$

Theorem 3 Let (H.1), (H.2) hold. Suppose further that

(H.3) $0<\Gamma<1$ for $\Gamma=\min \left\{\Gamma_{1}, \Gamma_{2}\right\}$, where

$$
\Gamma_{1}=-\beta^{M 2} \Phi_{2}^{2}-\gamma^{M}-\gamma^{M} \beta^{M} \Phi_{2}-2 \beta^{M} \Phi_{1}-v^{M 2} x^{* 2}+v^{M} x^{*}\left(\mu^{m}-1\right)
$$

and

$$
\Gamma_{2}=-\gamma^{M 2}-\gamma^{M} \beta^{M} \Phi_{2}-\gamma^{M}+\mu^{m}\left(2-\mu^{M}\right)-v^{M} x^{*}\left(1-\mu^{m}\right) .
$$


Then, there exists a unique uniformly asymptotically stable almost periodic solution $X=$ $(x(n), u(n))$ of system (4) which satisfies $x^{*} \leq x(n) \leq x^{*}$ and $u^{*} \leq u(n) \leq u^{*}$ for all $n \in \mathbb{Z}^{+}$.

Proof Let $p(n)=\ln x(n)$. In view of system (4), we have

$$
\left\{\begin{array}{l}
p(n+1)=p(n)+\beta(n) \ln ^{r}\left(\frac{K(n)}{x(n)}\right)-\alpha(n)-\gamma(n) u(n), \\
\Delta u(n)=-\mu(n) u(n)+v(n) e^{p(n)}
\end{array}\right.
$$

By the result of Theorem 2, it follows that system (13) has a bounded solution $(p(n), u(n))$ satisfying

$$
\ln x * \leq p(n) \leq \ln x^{*} \quad \text { and } \quad u^{*} \leq u(n) \leq u^{*}, \quad \text { for all } n \in \mathbb{Z}^{+} .
$$

Hence, $|p(n)| \leq \sigma$ and $|u(n)| \leq \rho$, where $\sigma=\max \left\{\left|\ln x_{*}\right|,\left|\ln x^{*}\right|\right\}$ and $\rho=\max \left\{u^{*}, u^{*}\right\}$.

For $(p, u) \in \mathbb{R}^{2}$, we define the norm $\|(p, u)\|=|p|+|u|$. Suppose that $X=(p(n), u(n))$ and $Y=(q(n), v(n))$ are any two solutions of system (13) defined on $\mathbb{Z}^{+} \times \Omega^{*} \times \Omega^{\prime \prime}$, then $\|X\| \leq \kappa$ and $\|Y\| \leq \kappa$ where $\kappa=\sigma+\rho$ and $\Omega^{*}=\left\{(p(n), u(n)) \in \mathbb{R}^{2}: \ln x^{*} \leq p(n) \leq \ln x^{*}, u^{*} \leq u(n) \leq\right.$ $u^{*}$, for all $n \in \mathbb{Z}^{+}$.

Consider the product system of (13)

$$
\left\{\begin{array}{l}
p(n+1)=p(n)+\beta(n) \ln ^{r}\left(\frac{K(n)}{e^{p(n)}}\right)-\alpha(n)-\gamma(n) u(n), \\
\Delta u(n)=-\mu(n) u(n)+v(n) e^{p(n)} \\
q(n+1)=q(n)+\beta(n) \ln ^{r}\left(\frac{K(n)}{e^{q(n)}}\right)-\alpha(n)-\gamma(n) v(n), \\
\Delta v(n)=-\mu(n) v(n)+v(n) e^{q(n)}
\end{array}\right.
$$

Construct a Lyapunov function defined on $\mathbb{Z}^{+} \times \Omega^{*} \times \Omega^{*}$ as follows:

$$
V(n, X, Y)=(p(n)-q(n))^{2}+(u(n)-v(n))^{2} .
$$

It is easy to see that the norm $\|X-Y\|=|p(n)-q(n)|+|u(n)-v(n)|$ and the norm $\|X-Y\|_{*}=$ $\left\{(p(n)-q(n))^{2}+(u(n)-v(n))^{2}\right\}^{\frac{1}{2}}$ are equivalent, that is, there exist two constants $C_{1}>0$ and $C_{2}>0$ such that

$$
C_{1}\|X-Y\| \leq\|X-Y\|_{*} \leq C_{2}\|X-Y\| .
$$

Thus

$$
\left(C_{1}\|X-Y\|\right)^{2} \leq\|X-Y\|_{*}^{2} \leq\left(C_{2}\|X-Y\|\right)^{2}
$$

or

$$
\left(C_{1}\|X-Y\|\right)^{2} \leq V(n, X, Y) \leq\left(C_{2}\|X-Y\|\right)^{2} .
$$

Let $a, b \in C\left(\mathbb{R}^{+}, \mathbb{R}^{+}\right)$such that $a(x)=C_{1}^{2} x^{2}$ and $b(x)=C_{2}^{2} x^{2}$. Thus, condition (i) of Lemma 2 is satisfied. 
Moreover,

$$
\begin{aligned}
&|V(n, X, Y)-V(n, \tilde{X}, \tilde{Y})| \\
&=\left|(p(n)-q(n))^{2}+(u(n)-v(n))^{2}-(\tilde{p}(n)-\tilde{q}(n))^{2}-(\tilde{u}(n)-\tilde{v}(n))^{2}\right| \\
& \leq\left|(p(n)-q(n))^{2}-(\tilde{p}(n)-\tilde{q}(n))^{2}\right|+\left|(u(n)-v(n))^{2}-(\tilde{u}(n)-\tilde{v}(n))^{2}\right| \\
&=|(p(n)-q(n))+(\tilde{p}(n)-\tilde{q}(n))||(p(n)-q(n))-(\tilde{p}(n)-\tilde{q}(n))| \\
& \quad+|(u(n)-v(n))+(\tilde{u}(n)-\tilde{v}(n))||(u(n)-v(n))-(\tilde{u}(n)-\tilde{v}(n))| \\
& \leq(|p(n)|+|q(n)|+|\tilde{p}(n)|+|\tilde{q}(n)|)(|p(n)-\tilde{p}(n)|+|q(n)-\tilde{q}(n)|) \\
&+(|u(n)|+|v(n)|+|\tilde{u}(n)|+|\tilde{v}(n)|)(|u(n)-\tilde{u}(n)|+|v(n)-\tilde{v}(n)|) \\
& \leq \Psi\{(|p(n)-\tilde{p}(n)|+|u(n)-\tilde{u}(n)|)+(|v(n)-\tilde{v}(n)|+|q(n)-\tilde{q}(n)|)\} \\
&= \Psi\{\|X-\tilde{X}\|+\|Y-\tilde{Y}\|\},
\end{aligned}
$$

where $\tilde{X}=(\tilde{p}(n), \tilde{u}(n)), \tilde{Y}=(\tilde{q}(n), \tilde{v}(n))$ and $\Psi=4 \max \{\sigma, \rho\}$. Therefore, condition (ii) of Lemma 2 is satisfied.

Finally, we calculate the difference $\Delta V(n, X, Y)$ along system (14). Indeed,

$$
\begin{aligned}
\Delta V_{(14)}(n, X, Y)= & V(n+1, X, Y)-V(n, X, Y) \\
= & (p(n+1)-q(n+1))^{2}-(p(n)-q(n))^{2} \\
& +(u(n+1)-v(n+1))^{2}-(u(n)-v(n))^{2} .
\end{aligned}
$$

In view of system (15), we observe that

$$
\begin{aligned}
(p(n+1)-q(n+1))^{2}= & {\left[(p(n)-q(n))+\beta(n) \ln ^{r}\left(\frac{K(n)}{e^{p(n)}}\right)\right.} \\
& \left.-\beta(n) \ln ^{r}\left(\frac{K(n)}{e^{q(n)}}\right)-\gamma(n)(u(n)-v(n))\right]^{2}
\end{aligned}
$$

or

$$
\begin{aligned}
(p(n+1)-q(n+1))^{2}= & (p(n)-q(n))^{2} \\
& +\left[\beta(n) \ln ^{r}\left(\frac{K(n)}{e^{p(n)}}\right)-\beta(n) \ln ^{r}\left(\frac{K(n)}{e^{q(n)}}\right)-\gamma(n)(u(n)-v(n))\right]^{2} \\
& +2(p(n)-q(n))\left[\beta(n) \ln ^{r}\left(\frac{K(n)}{e^{p(n)}}\right)-\beta(n) \ln ^{r}\left(\frac{K(n)}{e^{q(n)}}\right)\right. \\
& -\gamma(n)(u(n)-v(n))] .
\end{aligned}
$$

Thus,

$$
\begin{aligned}
(p(n+1)-q(n+1))^{2}= & (p(n)-q(n))^{2}+\left[\beta(n) \ln ^{r}\left(\frac{K(n)}{e^{p(n)}}\right)-\beta(n) \ln ^{r}\left(\frac{K(n)}{e^{q(n)}}\right)\right]^{2} \\
& +\gamma^{2}(n)(u(n)-v(n))^{2}
\end{aligned}
$$




$$
\begin{aligned}
& -2 \gamma(n)(u(n)-v(n))\left[\beta(n) \ln ^{r}\left(\frac{K(n)}{e^{p(n)}}\right)-\beta(n) \ln ^{r}\left(\frac{K(n)}{e^{q(n)}}\right)\right] \\
& +2(p(n)-q(n))\left[\beta(n) \ln ^{r}\left(\frac{K(n)}{e^{p(n)}}\right)-\beta(n) \ln ^{r}\left(\frac{K(n)}{e^{q(n)}}\right)\right] \\
& -2 \gamma(n)(p(n)-q(n))(u(n)-v(n)) .
\end{aligned}
$$

Moreover,

$$
\begin{aligned}
(u(n+1)-v(n+1))^{2} & =\left[(1-\mu(n)) u(n)+v(n) e^{p(n)}-(1-\mu(n)) v(n)-v(n) e^{q(n)}\right]^{2} \\
& =\left[(1-\mu(n))(u(n)-v(n))+v(n)\left(e^{p(n)}-e^{q(n)}\right)\right]^{2}
\end{aligned}
$$

or

$$
\begin{aligned}
(u(n+1)-v(n+1))^{2}= & (u(n)-v(n))^{2}+\mu(n)(\mu(n)-2)(u(n)-v(n))^{2} \\
& +v^{2}(n)\left(e^{p(n)}-e^{q(n)}\right)^{2} \\
& +2 v(n)(1-\mu(n))(u(n)-v(n))\left(e^{p(n)}-e^{q(n)}\right) .
\end{aligned}
$$

Substituting (16) and (17) back in (15), we obtain

$$
\begin{aligned}
\Delta V(n, X, Y)= & {\left[\beta(n) \ln ^{r}\left(\frac{K(n)}{e^{p(n)}}\right)-\beta(n) \ln ^{r}\left(\frac{K(n)}{e^{q(n)}}\right)\right]^{2}+\gamma^{2}(n)(u(n)-v(n))^{2} } \\
& -2 \gamma(n)(u(n)-v(n))\left[\beta(n) \ln ^{r}\left(\frac{K(n)}{e^{p(n)}}\right)-\beta(n) \ln ^{r}\left(\frac{K(n)}{e^{q(n)}}\right)\right] \\
& +2(p(n)-q(n))\left[\beta(n) \ln ^{r}\left(\frac{K(n)}{e^{p(n)}}\right)-\beta(n) \ln ^{r}\left(\frac{K(n)}{e^{q(n)}}\right)\right] \\
& -2 \gamma(n)(p(n)-q(n))(u(n)-v(n)) \\
& +\mu(n)(\mu(n)-2)(u(n)-v(n))^{2}+v^{2}(n)\left(e^{p(n)}-e^{q(n)}\right)^{2} \\
& +2 v(n)(1-\mu(n))(u(n)-v(n))\left(e^{p(n)}-e^{q(n)}\right) .
\end{aligned}
$$

By applying the Mean Value Theorem, we have

$$
\ln ^{r}\left(\frac{K(n)}{e^{p(n)}}\right)-\ln ^{r}\left(\frac{K(n)}{e^{q(n)}}\right)=-r\left[\ln \left(\frac{K(n)}{e^{\xi(n)}}\right)\right]^{r-1}(p(n)-q(n))
$$

and

$$
e^{p(n)}-e^{q(n)}=e^{\eta(n)}(p(n)-q(n)),
$$

where $\xi(n), \eta(n)$ lie between $p(n)$ and $q(n)$. Substituting (19) and (20) back in (18), we get

$$
\Delta V(n, X, Y)=\Omega_{1}+\Omega_{2}+\Omega_{3}+\Omega_{4}+\Omega_{5}+\Omega_{6}+\Omega_{7}+\Omega_{8},
$$

where

$$
\Omega_{1}(n)=\beta^{2}(n)\left(-r\left[\ln \left(\frac{K(n)}{e^{\xi(n)}}\right)\right]^{r-1}\right)^{2}(p(n)-q(n))^{2}, \quad \Omega_{2}(n)=\gamma^{2}(n)(u(n)-v(n))^{2},
$$




$$
\begin{aligned}
& \Omega_{3}(n)=-2 \gamma(n)(u(n)-v(n))\left(-r \beta(n) \ln ^{r-1}\left(\frac{K(n)}{e^{\xi(n)}}\right)\right)(p(n)-q(n)), \\
& \Omega_{4}(n)=2 \beta(n)(p(n)-q(n))^{2}\left(-r \ln ^{r-1}\left(\frac{K(n)}{e^{\xi(n)}}\right)\right), \\
& \Omega_{5}(n)=-2 \gamma(n)(p(n)-q(n))(u(n)-v(n)), \\
& \Omega_{6}(n)=\mu(n)(\mu(n)-2)(u(n)-v(n))^{2}, \quad \Omega_{7}(n)=v^{2}(n) e^{2 \eta(n)}(p(n)-q(n))^{2},
\end{aligned}
$$

and

$$
\Omega_{8}(n)=2 v(n) e^{\eta(n)}(1-\mu(n))(u(n)-v(n))(p(n)-q(n)) .
$$

By virtue of (H.1), (11) and (12), we observe that

$$
\begin{aligned}
\Omega_{1}(n) & \leq \beta^{M 2} \Phi_{2}^{2}(p(n)-q(n))^{2} \\
\Omega_{2}(n) & \leq \gamma^{M 2}(u(n)-v(n))^{2} \\
\Omega_{3}(n) & \leq 2 \gamma^{M} \beta^{M} \Phi_{2}|(u(n)-v(n))(p(n)-q(n))| \\
& \leq \gamma^{M} \beta^{M} \Phi_{2}(p(n)-q(n))^{2}+\gamma^{M} \beta^{M} \Phi_{2}(u(n)-v(n))^{2}, \\
\Omega_{4}(n) & \leq 2 \beta^{M} \Phi_{1}(p(n)-q(n))^{2} \\
\Omega_{5}(n) & \leq 2 \gamma^{M}|(p(n)-q(n))(u(n)-v(n))| \\
& \leq \gamma^{M}(p(n)-q(n))^{2}+\gamma^{M}(u(n)-v(n))^{2}, \\
\Omega_{6}(n) & \leq \mu^{m}\left(\mu^{M}-2\right)(u(n)-v(n))^{2}, \\
\Omega_{7}(n) & \leq v^{M 2} x^{* 2}(p(n)-q(n))^{2}, \\
\Omega_{8}(n) & \leq 2 v(n) e^{\eta(n)}(1-\mu(n))|(u(n)-v(n))(p(n)-q(n))| \\
& \leq 2 v^{M} x^{*}\left(1-\mu^{m}\right)|(u(n)-v(n))(p(n)-q(n))| \\
& \leq v^{M} x^{*}\left(1-\mu^{m}\right)(u(n)-v(n))^{2}+v^{M} x^{*}\left(1-\mu^{m}\right)(p(n)-q(n))^{2} .
\end{aligned}
$$

Substituting (22)-(29) back in (21), we obtain

$$
\begin{aligned}
\Delta V(n, X, Y) \leq & \beta^{M 2} \Phi_{2}^{2}(p(n)-q(n))^{2} \\
& +\gamma^{M 2}(u(n)-v(n))^{2} \\
& +\gamma^{M} \beta^{M} \Phi_{2}(p(n)-q(n))^{2}+\gamma^{M} \beta^{M} \Phi_{2}(u(n)-v(n))^{2} \\
& +2 \beta^{M} \Phi_{1}(p(n)-q(n))^{2} \\
& +\gamma^{M}(p(n)-q(n))^{2}+\gamma^{M}(u(n)-v(n))^{2} \\
& +\mu^{m}\left(\mu^{M}-2\right)(u(n)-v(n))^{2} \\
& +v^{M 2} x^{* 2}(p(n)-q(n))^{2} \\
& +v^{M} x^{*}\left(1-\mu^{m}\right)(u(n)-v(n))^{2}+v^{M} x^{*}\left(1-\mu^{m}\right)(p(n)-q(n))^{2} \\
= & -\left[-\beta^{M 2} \Phi_{2}^{2}-\gamma^{M}-\gamma^{M} \beta^{M} \Phi_{2}\right.
\end{aligned}
$$




$$
\begin{aligned}
& \left.-2 \beta^{M} \Phi_{1}-v^{M 2} x^{* 2}+v^{M} x^{*}\left(\mu^{m}-1\right)\right](p(n)-q(n))^{2} \\
& -\left[-\gamma^{M 2}-\gamma^{M} \beta^{M} \Phi_{2}-\gamma^{M}\right. \\
& \left.+\mu^{m}\left(2-\mu^{M}\right)-v^{M} x^{*}\left(1-\mu^{m}\right)\right](u(n)-v(n))^{2} \\
= & -\Gamma\left\{(p(n)-q(n))^{2}+(u(n)-v(n))^{2}\right\} \\
= & -\Gamma V(n, X, Y) .
\end{aligned}
$$

By virtue of the condition that $0<\Gamma<1$, assumption (iii) of Lemma 2 is satisfied. Thus, we conclude that there exists a unique uniformly asymptotically stable almost periodic solution $X=(p(n), u(n))$ of system (13) which satisfies $\ln x^{*} \leq p(n) \leq \ln x^{*}$ and $u^{*} \leq u(n) \leq$ $u^{*}$ for all $n \in \mathbb{Z}^{+}$. It follows that there exists a unique uniformly asymptotically stable almost periodic solution $X=(x(n), u(n))$ of system (4) which satisfies $x^{*} \leq x(n) \leq x^{*}$ and $u^{*} \leq$ $u(n) \leq u^{*}$ for all $n \in \mathbb{Z}^{+}$.

Assume the following condition:

(H.4) $\{\beta(n)\},\{K(n)\},\{\alpha(n)\},\{\gamma(n)\},\{\mu(n)\}$ and $\{v(n)\}$ are bounded nonnegative periodic sequences of period $\omega$.

Corollary 1 Let (H.2)-(H.4) hold. Then system (4) has a unique uniformly asymptotically stable periodic solution of period $\omega$.

\section{Some examples}

Example 1 Consider the following system:

$$
\left\{\begin{array}{l}
x(n+1)=x(n) \exp \left\{(0.25-0.05 \cos n) \ln ^{\frac{1}{3}}\left(\frac{1}{x(n)}\right)-0.2-0.2 u(n)\right\} \\
\Delta u(n)=-(0.35-0.05 \sin \pi n) u(n)+(0.4) x(n)
\end{array}\right.
$$

where $\beta(n)=0.25-0.05 \cos n, \alpha(n)=0.2, \gamma(n)=0.2, \mu(n)=0.35-0.05 \sin \pi n, v(n)=0.4$, $K(n) \equiv 1, r=\frac{1}{3}$. By calculation, we find $0.044 \approx x_{*}<x^{*} \approx 0.0498,0.044 \approx u_{*}<u^{*} \approx$ 0.0664 and $\Gamma_{1} \approx 0.8166, \Gamma_{2} \approx 0.2260$. Therefore, $\Gamma \approx 0.2260$. One can easily check the validity of conditions (H.1)-(H.3). Thus, by Theorem 1 and Theorem 3, system (30) is persistent and has a unique uniformly asymptotically stable almost periodic solution.

Example 2 Consider the following system:

$$
\left\{\begin{aligned}
x(n+1)= & x(n) \exp \left\{(0.35-0.05 \sin \sqrt{3} n) \ln \left(\frac{1+0.01 \sin \sqrt{3} n}{x(n)}\right)\right. \\
& -(0.2+0.01 \sin \sqrt{3} n)-(0.0015+0.0005 \sin \sqrt{3} n) u(n)\}, \\
\Delta u(n)= & -(0.35-0.05 \cos \sqrt{3} n) u(n)+(0.0015+0.0005 \sin \sqrt{3} n) x(n),
\end{aligned}\right.
$$

where $\beta(n)=0.35-0.05 \sin \sqrt{3} n, \alpha(n)=0.2+0.01 \sin \sqrt{3} n, \gamma(n)=0.0015+0.0005 \times$ $\sin \sqrt{3} n, \mu(n)=0.35-0.05 \cos \sqrt{3} n, \quad v(n)=0.0015+0.0005 \sin \sqrt{3} n, K(n)=1+$ $0.01 \sin \sqrt{3} n, r=2$. By calculation, we find $0.05823 \approx x^{*}<x^{*} \approx 0.73588,0.00145 \approx u^{*}<$ $u^{*} \approx 0.0049$ and $\Gamma_{1} \approx 0.267226, \Gamma_{2} \approx 0.22317$. Therefore, $\Gamma \approx 0.22317$. One can easily check the validity of conditions (H.1)-(H.3). Thus, by Theorem 1 and Theorem 3, system (31) is persistent and has a unique uniformly asymptotically stable almost periodic solution. 
Example 3 Consider the following system:

$$
\left\{\begin{aligned}
x(n+1)= & x(n) \exp \left\{(0.25-0.05 \sin n) \ln \left(\frac{1}{x(n)}\right)\right. \\
& -(0.35+0.05 \sin \pi n)-(0.001) u(n)\}, \\
\Delta u(n)= & -(0.25-0.05 \cos n) u(n)+(0.02) x(n),
\end{aligned}\right.
$$

where $\beta(n)=0.25-0.05 \sin n, \alpha(n)=0.35+0.05 \sin \pi n, \gamma(n)=0.001, \mu(n)=0.25-$ $0.05 \cos n, v(n)=0.02, K(n) \equiv 1, r \equiv 1$. By calculation, we find $0.12246 \approx x_{*}^{*}<x^{*} \approx 0.223$, $0.008164 \approx u *<u^{*} \approx 0.0223$ and $\Gamma_{1} \approx 0.596, \Gamma_{2} \approx 0.335$. Therefore, $\Gamma \approx 0.335$. One can easily check the validity of conditions (H.2)-(H.4). Thus, by Theorem 1 and Corollary 1 , system (32) is persistent and has a unique uniformly asymptotically stable periodic solution.

\section{Competing interests}

The author declares that he has no competing interests.

\section{Acknowledgements}

The author would like to express his sincere thanks to the editor Prof. Dr. Elena Braverman for handling the paper during the reviewing process and to the referees for suggesting some corrections that helped making the contents of the paper more accurate.

Received: 16 April 2012 Accepted: 24 August 2012 Published: 11 September 2012

\section{References}

1. Brauer, F, Castillo-Chavez, C: Mathematical Models in Population Biology and Epidemiology. Springer, Berlin (2001)

2. Edelstein-Keshet, L: Mathematical Models in Biology. Classics in Applied Mathematics, vol. 46. SIAM, Philadelphia (2004)

3. Jensen, A: Harvest in a fluctuating environment and conservative harvest for the Fox surplus production model. Ecol. Model. 182, 1-9 (2005)

4. Tsoularis, A, Wallace, J: Analysis of logistic growth models. Math. Biosci. 179(1), 21-55 (2002)

5. Brauer, F: Periodic environment and periodic harvesting. Nat. Resour. Model. 16, 233-244 (2003)

6. Fox, W: An exponential surplus-yield model for optimizing exploited fish populations. Trans. Am. Fish. Soc. 99, 80-88 (1970)

7. Kozusko, F, Bajzer, Z: Combining Gompartzian growth and cell population dynamics. Math. Biosci. 185(2), 153-167 (2003)

8. Cushing, JM, Costantino, RF, Dennis, B, Desharnais, RA, Henson, SM: Nonlinear population dynamics: models, experiments and data. J. Theor. Biol. 194, 1-9 (1998)

9. Berezanskya, L, Idels, L: Periodic Fox production harvesting models with delay. Appl. Math. Comput. 195(1), 142-153 (2008)

10. Idels, L: Stability analysis of periodic Fox production model. Can. Appl. Math. Q. 14(3), 331-341 (2006)

11. Alzabut, JO, Obaidat, S: Almost periodic solutions for Fox production harvesting model with delay. Electron. J. Qual. Theory Differ. Equ. 34, 1-12 (2012)

12. Gopalsamy, K, Weng, PX: Feedback regulation of logistic growth. Int. J. Math. Math. Sci. 16, 177-192 (1993)

13. Wang, Z, Li, YK: Almost periodic solutions of a discrete mutualism model with feedback controls. Discrete Dyn. Nat. Soc. 2010, Article ID 28603 (2010)

14. Chen, X, Fengde, C: Stable periodic solution of a discrete periodic Lotka-Volterra competition system with a feedback control. Appl. Math. Comput. 181, 1446-1454 (2006)

15. Nie, LF, Peng, JG, Teng, ZD: Permanence and stability in multispecies nonautonomous Lotka-Volterra competitive systems with delays and feedback controls. Math. Comput. Model. 49, 295-306 (2009)

16. Chen, XX: Almost periodic solutions of nonlinear delay population equation with feedback control. Nonlinear Anal., Real World Appl. 8, 62-72 (2007)

17. Chen, X, Fengde, C: Stable periodic solution of a discrete periodic Lotka-Volterra competition system with a feedback control. Appl. Math. Comput. 181(2), 1446-1454 (2006)

18. Agarwal, RP, Wong, PJY: Advanced Topics in Difference Equations. Kluwer Academic, Dordrecht (1997)

19. Agarwal, RP: Difference Equations and Inequalities, Theory, Methods and Applications, 2nd edn. Marcel Dekker, New York (2000)

20. Elayadi, S: An Introduction to Difference Equations, 3rd edn. Springer, New York (2005)

21. Gopalsamy, K: Stability and Oscillation in Delay Differential Equations of Population Dynamics: Mathematics and its Applications, vol. 74. Kluwer Academic, Dordrecht (1992)

22. Burton, TA: Stability and Periodic Solutions of Ordinary and Functional Differential Equations. Academic Press, San Diego (1985)

23. Bainov, D: Impulsive Differential Equations: Periodic Solutions and Applications. Longman, Harlow (1993) 
24. Wang, Q, Dai, B: Existence of positive periodic solutions for neutral population model with delays and impulse. Nonlinear Anal. 69, 3919-3930 (2008)

25. Liu, B, Huang, L: Existence and uniqueness of periodic solutions for a kind of Liénard equation with a deviating argument. Appl. Math. Lett. 21(1), 56-62 (2008)

26. Li, X, Yuan, Z: Existence of periodic solutions and closed invariant curves in a class of discrete-time cellular neural networks. Physica D, Nonlinear Phenom. 238(16), 1658-1667 (2009)

27. Zhao, W: New results of existence and stability of periodic solution for a delay multispecies logarithmic population model. Nonlinear Anal., Real World Appl. 10, 544-553 (2009)

28. Bereanu, C: Periodic solutions of some fourth-order nonlinear differential equations. Nonlinear Anal. 71, 53-57 (2009)

29. Liu, Z, Zhong, S, Yin, C, Chen, W: Permanence, extension and periodic solutions in a mathematical model of cell populations affected by periodic radiation. Appl. Math. Lett. 24, 1745-1750 (2011)

30. Besicovitch, AS: Almost Periodic Functions. Dover, New York (1954)

31. Fink, A: Almost Periodic Differential Equations. Lecture Notes in Mathematics, vol. 377. Springer, Berlin (1974)

32. He, CY: Almost Periodic Differential Equations. Higher Education, Beijing (1992)

33. Liu, YG, You, ZS, Cao, LP: On the almost periodic solution of cellular neural networks with distributed delays. IEEE Trans. Neural Netw. 18, 295-300 (2007)

34. Ahmad, S, Stamov, GT: Almost periodic solutions of $n$-dimensional impulsive competitive systems. Nonlinear Anal., Real World Appl. 10(3), 1846-1853 (2009)

35. Li, Z, Chen, F: Almost periodic solutions of a discrete almost periodic logistic equation. Math. Comput. Model. 50 254-259 (2009)

36. Luo, B: Travelling waves of a curvature flow in almost periodic media. J. Differ. Equ. 247, 2189-2208 (2009)

37. Huang, Z, Mohamad, S, Wang, X, Feng, C: Convergence analysis of general neural networks under almost periodic stimuli. Int. J. Circuit Theory Appl. 37, 723-750 (2009)

38. Alzabut, JO, Nieto, JJ, Stamov, GT: Existence and exponential stability of positive almost periodic solutions for a mode of hematopoiesis. Bound. Value Probl. 2009, Article ID 127510 (2009)

39. Yuan, R: On almost periodic solutions of logistic delay differential equations with almost periodic time dependence. J. Math. Anal. Appl. 330, 780-798 (2007)

40. Zhou, H, Zhou, Z, Wang, Q: Almost periodic solution for a class of Lasota-Wazewska model with infinite delays. Appl. Math. Comput. 218(8), 4501-4506 (2011)

41. Wu, W, Ye, Y: Existence and stability of almost periodic solutions of nonautonomous competitive systems with weak Allee effect and delays. Commun. Nonlinear Sci. Numer. Simul. 14(11), 3993-4002 (2009)

42. Stamov, GT, Petrov, N: Lyapunov-Razumikhin method for existence of almost periodic solutions of impulsive differential-difference equations. Nonlinear Stud. 15(2), 151-163 (2008)

43. Abbas, S, Bahuguna, D: Almost periodic solutions of neutral functional differential equations. Comput. Math. Appl. 55(11), 2539-2601 (2008)

44. Stamov, GT, Alzabut, JO, Atanasov, P, Stamov, AG: Almost periodic solutions for impulsive delay model of price fluctuations in commodity markets. Nonlinear Anal., Real World Appl. 12(6), 3170-3176 (2011)

45. Wang, YH, Xia, YH: The existence of almost periodic solutions of a certain nonlinear system. Commun. Nonlinear Sci. Numer. Simul. 16(2), 1060-1072 (2011)

46. Zhang, $T, L i, Y, Y e, Y$ : Persistence and almost periodic solutions for a discrete fishing model with feedback control. Commun. Nonlinear Sci. Numer. Simul. 16(3), 1564-1573 (2011)

47. Geng, J, Xia, Y: Almost periodic solutions of a nonlinear ecological model. Commun. Nonlinear Sci. Numer. Simul. 16(6), 2575-2597 (2011)

48. Stamov, GT, Stamova, IM, Alzabut, JO: Existence of almost periodic solutions for strongly stable nonlinear impulsive differential-difference equations. Nonlinear Anal. Hybrid Syst. 6(1), 818-823 (2012)

49. Cheban, D, Mammana, C: Invariant manifolds, global attractors and almost periodic solutions of nonautonomous difference equations. Nonlinear Anal. 56(4), 465-484 (2004)

50. Zhang, SN: Existence of almost periodic solution for difference systems. Ann. Differ. Equ. 16(2), 184-206 (2000)

doi:10.1186/1687-1847-2012-157

Cite this article as: Alzabut: Dynamics of almost periodic solutions for a discrete Fox harvesting model with feedback control. Advances in Difference Equations 2012 2012:157.

\section{Submit your manuscript to a SpringerOpen ${ }^{\odot}$ journal and benefit from:}

- Convenient online submission

- Rigorous peer review

- Immediate publication on acceptance

- Open access: articles freely available online

- High visibility within the field

- Retaining the copyright to your article 